\title{
Regeneração Natural de Espécies Ameaçadas de Extinção em Áreas Experimentais na Amazônia Oriental
}

\author{
Jaqueline Macedo Gomes ${ }^{1}$, João Olegário Pereira de Carvalho ${ }^{2}$, Ademir Roberto Ruschel ${ }^{3}$, José Natalino Macedo Silva², \\ Edson Marcos Leal Soares Ramos², Tatiana da Cunha Castro², Nisângela Severino Lopes Costa ${ }^{1}$, \\ Francimary da Silva Carneiro ${ }^{2} \&$ Larissa Martins Barbosa D'arace ${ }^{2}$
}

Recebido em 10/05/2020 - Aceito em 05/02/2021

1 Universidade Estadual da Região Tocantina do Maranhão/UEMASUL. Brasil. <jaquelinemacedogomes@hotmail.com, nisangela.lopes@uemasul.edu.br>.

2 Universidade Federal Rural da Amazônia/UFRA. Brasil. <olegario.carvalho@gmail.com, silvanatalino734@gmail.com, ramosedson@gmail.com, ccastro.tatiana@gmail.com, francimarycarneiro@gmail.com, larissamartins350@gmail.com>.

3 Embrapa Amazônia Oriental. Brasil. <ademir.ruschel@embrapa.br>.

\begin{abstract}
RESUMO - Este trabalho avaliou as alterações ocorridas no número de indivíduos da regeneração natural de Hymenaea parvifolia Huber (Jutaí-mirim), Hymenolobium excelsum Ducke (Angelim-damata) e Vouacapoua americana Aubl. (Acapu) classificadas como ameaçadas de extinção pela União Internacional de Conservação da Natureza (IUCN), em três áreas experimentais de manejo florestal no estado do Pará. Determinou-se a abundância dessas espécies pela razão entre o número de indivíduos de cada espécie e a área da amostra em hectares; em seguida, foi realizada a estatística descritiva. Os resultados mostram que a ausência de indivíduos em regeneração natural de Hymenaea parvifolia e Hymenolobium excelsum na área não explorada e a baixa densidade na área manejada indicam a dificuldade que essas espécies têm para regenerar e se estabelecer na floresta. E isso pode leválas à extinção na floresta estudada. A regeneração natural de Vouacapoua americana é abundante, garantindo a sua permanência constante, sem risco de extinção na área em estudo, embora esteja incluída na lista de espécies ameaçadas.
\end{abstract}

Palavras-chave: Conservação de espécies; dinâmica em florestas naturais; Hymenaea parvifolia; Hymenolobium excelsum; Vouacapoua americana.

\section{Natural Regeneration of Endangered Species in Experimental Areas in the Eastern Amazon}

ABSTRACT - This work evaluated the changes in the number of individuals in the natural regeneration of Hymenaea parvifolia Huber (jutaí-mirim), Hymenolobium excelsum Ducke (angelim-da-mata) and Vouacapoua americana Aubl. (acapu) classified as endangered by the International Union for Nature Conservation (IUCN), in three experimental forest management areas in the State of Pará. The abundance in the populations of these species was determined by the ratio between the number of individuals of each species and the sample area in hectares. Descriptive statistics were performed. The results show that the absence of individuals in the natural regeneration of $H$. parvifolia and $H$. excelsum in the treatment with no logging and the low density of individuals in the treatments in the managed forest indicate the difficulty of these species to regenerate and establish themselves in the forest, which can lead to extinction in the area. The natural regeneration of $V$. americana is abundant in the area, guaranteeing its permanence, without risk of extinction.

Keywords: Conservation of species; dynamics in natural forests; Hymenaea parvifolia; Hymenolobium excelsum; Vouacapoua americana. 


\section{Regeneración Natural de Especies Amenazadas en Áreas Experimentales de la Amazonía Oriental}

RESUMEN - Este trabajo evaluó los cambios en el número de individuos en la regeneración natural de Hymenaea parvifolia Huber (Jutaí-mirim), Hymenolobium excelsum Ducke (Angelim-da-mata) y Vouacapoua americana Aubl. (Acapu) clasificada como en peligro por la Unión Internacional para la Conservación de la Naturaleza (UICN), en tres áreas de manejo forestal experimental en el estado de Pará. La abundancia de estas especies se determinó por la relación entre el número de individuos de cada especie y la muestra área en hectáreas, luego se realizaron estadísticas descriptivas. Los resultados muestran que la ausencia de individuos en regeneración natural de Hymenaea parvifolia y Hymenolobium excelsum en el área inexplorada y la baja densidad en el área manejada indican la dificultad que tienen estas especies para regenerarse y establecerse en el bosque. Esto puede llevar a la extinción del bosque estudiado. La regeneración natural de Vouacapoua americana es abundante, garantizando su permanencia constante, sin riesgo de extinción en el área de estudio, aunque está incluida en la lista de especies amenazadas.

Palabras clave: Conservación de especies; dinámica en bosques naturales; Hymenaea parvifolia; Hymenolobium excelsum; Vouacapoua americana.

\section{Introdução}

O aumento contínuo da população e as pressões econômicas resultam no desmatamento e na fragmentação da floresta. Para seu controle tem-se estimulado a adoção de tecnologias adequadas, como aquelas que são aplicadas em áreas de manejo florestal sustentável (Leite \& Resende, 2010). A viabilidade do manejo sustentável está em garantir um fluxo constante de madeira com potencial comercial (Braz et al., 2004), sem interromper de maneira irreversível os processos de sustentação do ecossistema (Higman et al., 2015).

As alterações nos ambientes florestais são necessárias para manter a dinâmica da floresta $e$ conservar as espécies que necessitam das clareiras para regenerar. A regeneração natural é um processo de sustentação das florestas tropicais, pois garante a sua renovação. Segundo Jardim (2015), muitos táxons têm poucos ou nenhum indivíduo em regeneração natural, mas isso não é um argumento válido para impedir a exploração, pois se os adultos existem há um mecanismo para isso, ou seja, tais espécies dependem da formação de clareiras.

A formação de clareiras gera alterações na floresta, cuja colonização é influenciada pelas respostas ecofisiológicas das espécies na área. $\mathrm{O}$ entendimento da dinâmica de clareiras em florestas tropicais é importante para a restauração da floresta, para o manejo sustentável e para a conservação dos remanescentes florestais (Martins et al., 2012).

As práticas de manejo florestal sustentável buscam minimizar os impactos negativos da exploração e imitar os processos naturais de abertura de clareiras em florestas, estimulando a regeneração natural de espécies que podem ser consideradas ameaçadas, exatamente pela falta de plântulas, mudas e varas. De acordo com Jardim (2015), a análise da regeneração natural é necessária e importante para determinar se esse processo é capaz de garantir que as espécies exploradas irão repor o material colhido, pois o conceito de sustentabilidade está baseado na capacidade da floresta se regenerar.

A análise da regeneração natural permite inferir sobre a dinâmica de comunidades e populações de espécies arbóreas que são fundamentais para o manejo sustentável. No entanto, entender o processo pelo qual as espécies arbóreas se regeneram é uma tarefa complexa, pois está relacionada às suas características ecológicas e às condições ambientais (Santos et al., 2015).

Diante do exposto, neste estudo avaliou-se a regeneração natural das espécies ameaçadas de extinção: Hymenaea parvifolia Huber (Jutaí-mirim), Hymenolobium excelsum Ducke (Angelim-da-mata) e Vouacapoua americana Aubl. (Acapu) com o intuito de garantir a conservação das mesmas em área de manejo florestal sustentável. 


\section{Material e Métodos}

\section{Áreas de estudo}

Floresta do Tapajós

As duas áreas experimentais na Floresta Nacional do Tapajós, município de Belterra, Pará, estão localizadas, à altura do $\mathrm{km} 67$ (02 ${ }^{\circ}$ 53'

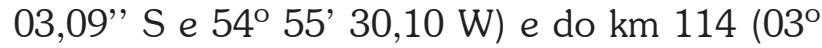
18 ' 32' - $03^{\circ} 19^{\prime} 21^{\prime \prime}$ S e $54^{\circ} 56^{\prime} 28^{\prime \prime}$ - 54' $56^{\prime}$ 15 " W) da BR 163, Rodovia Santarém-Cuiabá.

Os solos que predominam na região são do tipo Latossolo amarelo distrófico e argissolo amarelo distrófico (Oliveira Junior \& Correa, 2001). O clima da região é do tipo Am, segundo a classificação de Köppen-Geiger (Kottek et al., 2006), com temperatura média anual de $27^{\circ} \mathrm{C}$, umidade relativa do ar de $87 \%$ e precipitação pluviométrica média anual é de $1.982 \mathrm{~mm}$ (INMET, 2017). A vegetação da área de estudo é do tipo Floresta Ombrófila Densa (IBGE, 2012).

\section{Floresta do Moju}

O Campo Experimental da Embrapa Amazônia Oriental no município de Moju, estado do Pará, tem área total de 1.050ha, situado entre as coordenadas geográficas $02^{\circ} 08^{\prime} 14^{\prime \prime}$ e $02^{\circ} 12^{\prime}$ $26^{\prime \prime}$ de latitude Sul e $48^{\circ} 47^{\prime} 34^{\prime \prime}$ e $48^{\circ} 48^{\prime} 14^{\prime \prime}$ de longitude a Oeste de Greenwich (Lopes et al., 2001).

O clima da região é do tipo Af, segundo a classificação de Köppen-Geiger (Kottek, et al., 2006), considerando os dados coletados na estação meteorológica de Belém, a temperatura média anual é de $28^{\circ} \mathrm{C}$, umidade relativa do ar de $83 \%$ e a precipitação pluviométrica média anual é de $3.473 \mathrm{~mm}$ (INMET, 2017). O relevo varia de plano a suavemente ondulado e o solo predominante é do tipo Latossolo Amarelo distrófico (IBGE, 2008). A vegetação da área experimental é classificada como Floresta Ombrófila Densa (IBGE, 2012).

\section{Espécies}

As espécies Hymenaea parvifolia Huber, Hymenolobium excelsum Ducke e Vouacapoua americana Aubl. foram selecionadas para o estudo por terem sido exploradas nas áreas experimentais e por estarem contidas na lista vermelha da União Internacional de Conservação da Natureza (IUCN,
2012) e na lista de espécies ameaçadas de extinção do Ministério do Meio Ambiente (MMA, 2014). $H$. parvifolia e $H$. excelsum foram exploradas em 1979 na área experimental do km 67 e em 1982 na área do $\mathrm{km} 114$. V. americana foi explorada em 1997 na área do Campo Experimental do Moju.

H. parvifolia ou jutaí-mirim, pertence à família Fabaceae, possui ampla distribuição geográfica, ocorre nos estados do Acre, Amazonas, Pará, Rondônia, Roraima, Maranhão, Piauí e Mato Grosso (Fernandes et al., 2015). É uma espécie que possui árvores de grande porte, que chegam a atingir 1 metro de diâmetro e altura entre 25 e 40m (Salomão et al., 1995).

A floração ocorre no período de agosto a fevereiro e a frutificação durante o ano todo com a disseminação no período de agosto a março (Leão \& Carvalho, 2001). A dispersão é barocórica e zoocórica (Salomão et al., 2007) e a temperatura ótima para germinação da espécie está entre 25 e $35^{\circ} \mathrm{C}$ (Silva \& Cesarino, 2016).

H. parvifolia pertence ao grupo ecológico das espécies intermediárias (Jardim et al., 1996) e segundo Silva et al. (2007) possui melhores chances de regenerar sob dossel fechado. Sua madeira tem alto valor comercial e produz goma para a indústria e seus frutos servem de alimento para os humanos e para os animais (Salomão et al., 1995).

H. excelsum (angelim-da-mata, angelimrajado, fava-folha-fina) pertence à Fabaceae e ocorre nos estados do Amapá, Amazonas e Pará (Ferreira et al., 2004a). Sua floração tem início no mês de março e se estende até junho. A frutificação ocorre de maio a outubro $e$ a disseminação ocorre de dezembro a fevereiro (Vieira et al., 2002). Pertence ao grupo ecológico de espécies tolerantes à sombra (Lopes et al., 2001). Sua madeira é utilizada na construção em geral, tornearia, marcenaria, carpintaria, dormentes, tacos e objetos de adornos (Ferreira et al., 2004b).

V. americana (acapu) pertence à Fabaceae, ocorre nos estados do Amapá, Amazonas e Pará (Almeida et al., 2001). A floração e a frutificação ocorrem nos meses de janeiro a março e a disseminação acontece no período de junho a setembro (Leão et al., 2015). A dispersão é barocórica e zoocórica, feita por cutias (Desyprocta aguti L.) e pacas (Agouti paca L.) (Forget, 1990). Também pertence ao grupo ecológico das espécies 
vegetais tolerantes à sombra (Lopes et al., 2001). Sua madeira pode ser utilizada na carpintaria naval, objetos de decoração, tacos, móveis de fino acabamento, peças torneadas, acabamentos internos de iates (Gonzaga, 2006).

\section{Coleta de dados}

\section{Floresta do Tapajós}

Foram instaladas e medidas 48 parcelas permanentes na área do $\mathrm{km} 114$ e 36 parcelas permanentes no km 67 da Floresta Nacional do Tapajós. As parcelas permanentes possuem uma área de 0,25 ha $(50 \mathrm{~m} \times 50 \mathrm{~m})$ cada. Para facilitar a coleta de dados cada parcela foi dividida em 25 subparcelas de $10 \mathrm{~m} \times 10 \mathrm{~m}$, onde foram inventariadas todas as árvores com DAP $\geq 5 \mathrm{~cm}$. Dentre essas 25 subparcelas de $10 \mathrm{~m} \times 10 \mathrm{~m}$, foram sorteadas cinco, no centro das quais foram estabelecidas parcelas de $5 \mathrm{~m} \times 5 \mathrm{~m}$ para medição de varas (indivíduos com $2,5 \mathrm{~cm}<\mathrm{DAP}<$ $5,0 \mathrm{~cm})$. Cada parcela de $5 \mathrm{~m} \times 5 \mathrm{~m}$ foi subdividida em quatro triângulos iguais de $6,25 \mathrm{~m}^{2}$, dentre os quais foi sorteado um para inventariar as mudas (indivíduos com altura superior a $30 \mathrm{~cm}$ e diâmetro inferior a $2,5 \mathrm{~cm}$ ). Assim, as varas foram monitoradas em 240 parcelas permanentes de $5 \mathrm{~m}$ x $5 \mathrm{~m}$ (área amostral de 0,6ha) e as mudas foram monitoradas em 240 parcelas triangulares de 6,25 $\mathrm{m} 2$ de dimensões 5,00m x 3,54m x 3,54m (área amostral de 0,15ha) (Carvalho, 1992). Para a presente pesquisa foram considerados os dados coletados na classe de varas e de mudas.

Para a área do $\mathrm{km} 67$ os dados foram coletados todos após a exploração florestal nos anos de 1981, 1982, 1983, 1985, 1987, 1992, 1997, 2007, 2012, 2014 e 2015. E para a área do $\mathrm{km} 114$ os dados foram coletados antes da exploração florestal em 1981 e após a exploração em 1983, 1987, 1989, 1995, 2003, 2008 e 2012. No Km 114, no período de 1993-1994 foram aplicados tratamentos silviculturais de desbaste sistemático por meio da anelagem por entalhes, os tratamentos estão descritos em Oliveira et al. (2006).

\section{Floresta do Moju}

Em 1998 foram instaladas 117 parcelas permanentes em 9 clareiras para o monitoramento de varas e mudas. No centro de cada clareira foi instalada uma parcela de $2 \mathrm{~m} \times 2 \mathrm{~m}$, outra na borda e outras a $20 \mathrm{~m}$ e $40 \mathrm{~m}$ da borda nas direções norte, sul, leste e oeste (Figura 1). A amostra para medição das varas e mudas foi de 0,047 ha.

As parcelas foram monitoradas trimestralmente no período de junho de 1998 a junho de 2001; em 2007, foram feitas duas avaliações e uma no ano de 2010, totalizando 16 medições em 12 anos (Jardim \& Quadros, 2016; Quadros, 2012).

\section{Análise dos dados}

A regeneração natural das populações das três espécies foi avaliada por meio da abundância (A), que é determinada pela razão entre o número de indivíduos da espécie em questão $\left(\mathrm{n}_{\mathrm{i}}\right)$ e a área da amostra geralmente dada em hectares (a) $\left(A=n_{i} / a\right)$. Embora as parcelas no km 114 tenham sido medidas oito vezes, as parcelas no $\mathrm{km} 67$ medidas onze vezes e as do Moju 16 vezes, foram escolhidas cinco medições em cada área, ao longo do período monitorado, para serem avaliadas (Tabela 1). Foi realizada a estatística descritiva.

\section{Resultados e Discussão Hymenaea parvifolia Huber}

Na Tabela 2 é apresentada a abundância dos indivíduos de $H$. parvifolia nas áreas experimentais do $\mathrm{km} 114$ e km 67.

No km 114 não foi registrada a presença da espécie em regeneração natural antes da exploração (1981). Na categoria de varas, a espécie esteve presente apenas em 1983, com somente um indivíduo na amostra, equivalendo a duas varas por hectare. Na classe de mudas foram registrados 33 indivíduos por hectare, imediatamente após a exploração (1983), permanecendo na área até sete anos após a exploração (1989). Porém, em 1995, logo após os tratamentos silviculturais, não houve registro da espécie, que só voltou a ser encontrada no final do monitoramento (2012) com 7 mudas ha-1.

No km 67 nenhuma vara foi encontrada em todo o período estudado. Entretanto, a população de mudas foi muito dinâmica, pois quatro anos após a exploração (1983), foram encontradas 18 mudas por hectare. No entanto esse número foi reduzido pela metade aos oito 


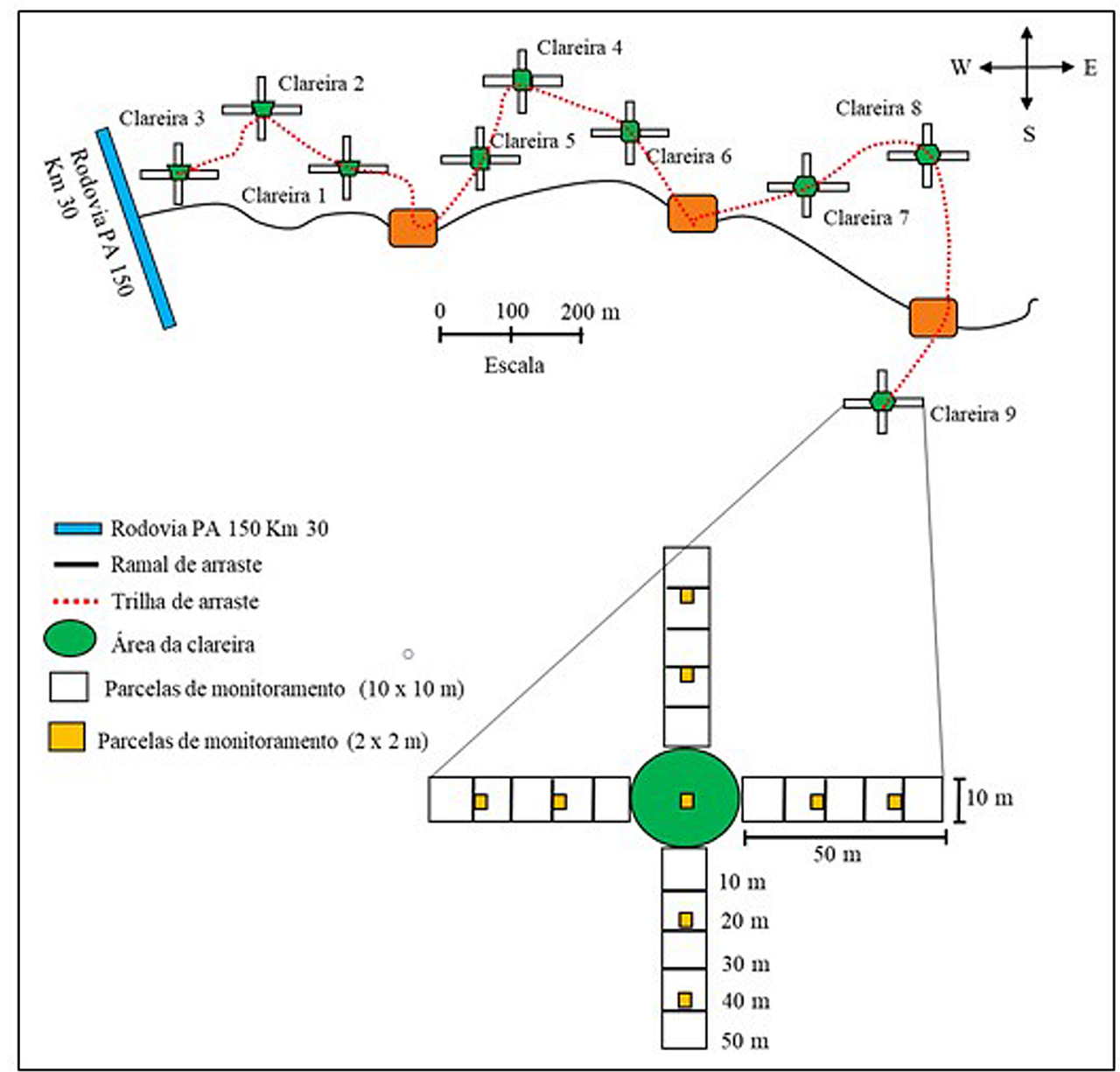

Fonte: Adaptado de Costa et al. (2020)

Figura 1 - Desenho esquemático das parcelas instaladas nas clareiras para o monitoramento da regeneração natural no campo experimental da Embrapa Amazônia Oriental, Moju-Pará.

Tabela 1 - Anos de medições nos quais foram coletados e avaliados os dados para o cálculo da abundância $e$ frequência da regeneração natural das três espécies, nas três áreas de estudo.

\begin{tabular}{|c|c|c|}
\hline $\mathbf{k m} \mathbf{1 1 4}$ & $\mathbf{k m} \mathbf{6 7}$ & $\mathbf{M o j u}$ \\
\hline 1981 & 1981 & 1998 \\
\hline 1983 & 1983 & 1999 \\
\hline 1989 & 1987 & 2000 \\
\hline 1995 & 1997 & 2007 \\
\hline
\end{tabular}

anos após a exploração (1987), desaparecendo da amostra em 1977. Contudo, ao final do período monitorado (2012), foram registradas nove mudas por hectare no local de estudo.
A regeneração natural da espécie no km 67 foi menor do que no $\mathrm{km} \mathrm{114,} \mathrm{sem} \mathrm{a} \mathrm{presença} \mathrm{de}$ varas e com poucas mudas, causando preocupação quanto à sua permanência na área. Isso indica que 
Tabela 2 - Número de indivíduos $(\mathrm{N})$ e Abundância $\left(\mathrm{A}=\right.$ número de indivíduos ha $\left.{ }^{-1}\right)$ de varas e mudas de Hymenaea parvifolia Huber em duas áreas experimentais (km 114 e km 67) administradas pela Embrapa Amazônia Oriental, localizadas na Floresta Nacional do Tapajós.

\begin{tabular}{|c|c|c|c|c|c|c|c|c|c|c|}
\hline \multirow[b]{3}{*}{ Categoria } & \multicolumn{5}{|c|}{ Km 114} & \multicolumn{5}{|c|}{ Km 67} \\
\hline & \multicolumn{5}{|c|}{ (amostra para varas $=0,6 \mathrm{ha}$; mudas $=0,15 \mathrm{ha}$ ) } & \multicolumn{5}{|c|}{ (amostra para varas $=0,45 \mathrm{ha} ;$ mudas $=0,11 \mathrm{ha})$} \\
\hline & Ano & $\mathbf{N}$ & A & $\mathbf{M}$ & $\mathbf{S}$ & Ano & $\mathbf{N}$ & A & $\mathbf{M}$ & $\mathbf{s}$ \\
\hline Vara & \multirow{2}{*}{1981} & 0 & 0 & 0 & 0 & \multirow{2}{*}{1981} & 0 & 0 & 0 & 0 \\
\hline Muda & & 0 & 0 & 0 & 0 & & 1 & 9 & 0,05 & 0,66 \\
\hline Vara & \multirow{2}{*}{1983} & 1 & 2 & 0,01 & 0,11 & \multirow{2}{*}{1983} & 0 & 0 & 0 & 0 \\
\hline Muda & & 5 & 33 & 0,14 & 0,95 & & 2 & 18 & 0,1 & 0,93 \\
\hline Vara & \multirow{2}{*}{1989} & 0 & 0 & 0 & 0 & \multirow{2}{*}{1987} & 0 & 0 & 0 & 0 \\
\hline Muda & & 5 & 33 & 0,14 & 0,95 & & 1 & 9 & 0,05 & 0,66 \\
\hline Vara & \multirow{2}{*}{1995} & 0 & 0 & 0 & 0 & \multirow{2}{*}{1997} & 0 & 0 & 0 & 0 \\
\hline Muda & & 0 & 0 & 0 & 0 & & 0 & 0 & 0 & 0 \\
\hline Vara & \multirow{2}{*}{2012} & 0 & 0 & 0 & 0 & \multirow{2}{*}{2012} & 0 & 0 & 0 & 0 \\
\hline Muda & & 1 & 6,7 & 0,03 & 0,43 & & 1 & 9 & 0,05 & 0,66 \\
\hline
\end{tabular}

M: média do número de árvores por hectare; $\mathrm{S}$ desvio padrão do número de árvores por hectare.

há necessidade de práticas silviculturais baseadas na ecologia da espécie, para estimular a sua regeneração.

A ausência na regeneração natural de uma determinada espécie, que ocorre na floresta, pode ser ocasionada pela deficiência de luminosidade na área. No entanto, de acordo com Silva et al. (2007), H. parvifolia apresentaria melhores chances de regenerar sob a cobertura da floresta, tolerando intensidades luminosas reduzidas. Porém, nesta pesquisa a espécie apresentou dificuldades para regenerar. No estudo de Oliveira (1997) em uma floresta secundária no município de Belterra, foi observada a presença de indivíduos adultos de $H$. parvifolia, mas não houve ocorrência de varas e mudas. Segundo Rabelo et al. (2000), o fato de algumas espécies não apresentarem regeneração natural pode estar relacionado a diversos fatores como: as ações antrópicas ocorridas na área; baixa abundância de indivíduos adultos; e predação. Dessa forma, são necessários estudos específicos relacionados à fenologia e dispersão de frutos $e$ sementes para melhor conhecer as estratégias adotadas pelas espécies para regenerar.
Na pesquisa de Ngo \& Hölscher (2014) foi observado que após a exploração os indivíduos adultosearegeneraçãodeespéciesrarasocorreram na floresta, porém em baixas densidades. Os autores advertem que a exploração tem um efeito adverso sobre a regeneração devido à diminuição do fornecimento de sementes na área.

Mostacedo \& Fredericksen (1999) afirmam que $H$. parvifolia possui regeneração natural escassa e seus mecanismos para regenerar são pouco compreendidos, dificultando o seu manejo. Comentam ainda que a regeneração bem sucedida talvez seja o único e mais importante passo no sentido de atingir a sustentabilidade em florestas manejadas.

\section{Hymenolobium excelsum Ducke}

Na Tabela 3 é apresentada a abundância dos indivíduos de $H$. excelsum nas áreas experimentais do $\mathrm{km} 114$ e km 67.

No km 114, não havia regeneração natural antes da exploração (1981). Logo após 
Tabela 3 - Número de indivíduos $(\mathrm{N})$ e Abundância $\left(\mathrm{A}=\right.$ número de indivíduos ha $\left.{ }^{-1}\right)$ de varas e mudas de Hymenolobium excelsum Ducke em duas áreas experimentais (km 114 e km 67) administradas pela Embrapa Amazônia Oriental, localizadas na Floresta Nacional do Tapajós.

\begin{tabular}{|c|c|c|c|c|c|c|c|c|c|c|}
\hline \multirow[b]{3}{*}{ Categoria } & \multicolumn{5}{|c|}{ Km 114} & \multicolumn{5}{|c|}{ Km 67} \\
\hline & \multicolumn{5}{|c|}{ (amostra para varas $=0,6 \mathrm{ha} ;$ mudas $=0,15 \mathrm{ha})$} & \multicolumn{5}{|c|}{ (amostra para varas $=0,45 \mathrm{ha} ;$ mudas $=0,11 \mathrm{ha})$} \\
\hline & Ano & $\mathbf{N}$ & A & $\mathbf{M}$ & $\mathbf{S}$ & Ano & $\mathbf{N}$ & A & $\mathbf{M}$ & $\mathbf{s}$ \\
\hline Vara & \multirow{2}{*}{1981} & 0 & 0 & 0 & 0 & \multirow{2}{*}{1981} & 1 & 2 & 0,01 & 0,17 \\
\hline Muda & & 0 & 0 & 0 & 0 & & 4 & 36 & 0,20 & 1,31 \\
\hline Vara & \multirow{2}{*}{1983} & 1 & 2 & 0,01 & 0,11 & \multirow{2}{*}{1983} & 0 & 0 & 0 & 0 \\
\hline Muda & & 1 & 7 & 0,03 & 0,43 & & 2 & 18 & 0,10 & 1,33 \\
\hline Vara & \multirow{2}{*}{1989} & 1 & 2 & 0,01 & 0,11 & \multirow{2}{*}{1987} & 0 & 0 & 0 & 0 \\
\hline Muda & & 0 & 0 & 0 & 0 & & 4 & 36 & 0,20 & 1,62 \\
\hline Vara & \multirow{2}{*}{1995} & 0 & 0 & 0 & 0 & \multirow{2}{*}{1997} & 0 & 0 & 0 & 0 \\
\hline Muda & & 0 & 0 & 0 & 0 & & 2 & 18 & 0,10 & 0,93 \\
\hline Vara & \multirow{2}{*}{2012} & 0 & 0 & 0 & 0 & \multirow{2}{*}{2012} & 0 & 0 & 0 & 0 \\
\hline Muda & & 1 & 7 & 0,03 & 0,43 & & 0 & 0 & 0 & 0 \\
\hline
\end{tabular}

M: média do número de árvores por hectare; S desvio padrão do número de árvores por hectare.

a exploração (1983) foram registradas tanto varas ( 2 varas ha $^{-1}$ ) quanto mudas ( 7 mudas ha $^{-1}$ ), entretanto as mudas morreram antes de 1989 e as varas morreram logo após a aplicação dos tratamentos silviculturais (1995). No final do monitoramento (2012), trinta anos após a exploração e dezoito anos após a aplicação dos tratamentos silviculturais, foi registrada novamente a presença de mudas ( 7 mudas ha ${ }^{-1}$ ) na área.

No km 67, a regeneração natural de $H$. excelsum esteve presente desde 1981, aos dois anos após a exploração (2 varas ha-1 e 36 mudas ha-1), mas logo a seguir (1983) não houve mais registro de varas $e$ as mudas foram reduzidas para a metade (18 mudas ha-1). Mesmo assim, continuaram presentes na área até vinte e oito anos após a exploração, porém no final do monitoramento (2012), trinta e três anos após a exploração, não foram encontradas varas e mudas da espécie na amostra.

A densidade das espécies avaliadas (Hymenaea parvifolia e Hymenolobium excelsum) pode indicar problemas futuros em relação à sua permanência na área, se a sua regeneração não conseguir se estabelecer. A aplicação de tratamentos silviculturais, como corte de cipós (Souza et al., 2014) e desbaste de liberação de copas, para favorecer a regeneração natural (Wadsworth \& Zweede, 2006), condução de mudas (Carvalho et al., 2013) e plantio nas clareiras resultantes da exploração florestal (Reis et al., 2014), são estratégias que podem garantir a conservação dessas espécies na área.

Após a exploração as espécies comerciais têm pouca capacidade para se regenerar e a manutenção de $10 \%$ dos indivíduos das espécies exploradas com DAP acima de $50 \mathrm{~cm}$, regra estabelecida pela legislação (MMA, 2006), não parece ser suficiente para garantir a regeneração natural dessas espécies (Schwartz et al., 2017).

Segundo Schwartz et al. (2013), quando a regeneração natural de espécies comerciais não é suficiente, o plantio em clareiras é recomendado para aumentar o número de indivíduos de espécies pouco frequentes. Schwartz et al. (2017) enfatizaram, que o sucesso do plantio visando à conservação de espécies comerciais de baixa densidade requer um suprimento de sementes de alta qualidade. Por isso, é necessário identificar e monitorar grupos geneticamente diversificados 
de árvores na área de manejo, para efetuar a coleta de sementes. Karsenty \& Gourlet-Fleury (2006) sugerem proteger áreas dentro da unidade de manejo para que se possa preservar habitat, para servir de refúgio para animais e ser fonte de sementes.

Na pesquisa de Muñoz et al. (2010) sobre a dinâmica da regeneração natural em clareiras em uma floresta tropical úmida no Peru, dentre as espécies encontradas, foi observado que $H$. excelsum se beneficiou do aumento da radiação solar originada pela abertura de clareiras. No presente estudo, os resultados mostram que a exploração não beneficiou as varas e mudas e não facilitou o seu estabelecimento.

As atividades de manejo florestal sustentável, por serem planejadas e bem executadas, podem-se constituir em estratégia para a conservação dessas espécies (Hymenaea parvifolia e
Hymenolobium excelsum) nas áreas manejadas. $\mathrm{O}$ monitoramento de suas populações, tanto de árvores como de varas e mudas, possibilita a avaliação $e$ análises de suas características ecológicas e do comportamento silvicultural, assim como das características edafo-climáticas da área. Isso poderá facilitar as tomadas de decisões para evitar o declínio ou o desaparecimento dessas espécies da área. As atividades do manejo florestal sustentável, segundo Gourlet-Fleury et al. (2005), devem garantir, pelo menos, a capacidade de regeneração das espécies exploradas, em curto, médio e longo prazo.

\section{Vouacapoua americana Aubl.}

$\mathrm{Na}$ Tabela 4, é apresentada a abundância dos indivíduos de V. americana na área do Campo Experimental da Embrapa no município do Moju.

Tabela 4 - Número de indivíduos $(\mathrm{N})$ e Abundância $\left(\mathrm{A}=\right.$ número de indivíduos ha-1 ${ }^{-1}$ de varas e mudas de Vouacapoua americana Aubl. na área do Campo Experimental da Embrapa Amazônia Oriental no município de Moju, estado do Pará.

\begin{tabular}{|c|c|c|c|c|c|}
\hline Categoria & Ano & N & A & M & S \\
\hline Vara & & 1 & 21 & 0,18 & 1,98 \\
\hline Muda & 1998 & 19 & 406 & 3,47 & 17,29 \\
\hline Vara & & 1 & 21 & 0,18 & 1,98 \\
\hline Muda & 1999 & 25 & 534 & 4,57 & 26,59 \\
\hline Vara & & 2 & 43 & 0,37 & 2,78 \\
\hline Muda & 2000 & 25 & 534 & 4,57 & 26,59 \\
\hline Vara & & 2 & 43 & 0,37 & 2,78 \\
\hline Muda & 2007 & 6 & 128 & 1,10 & 4,73 \\
\hline Vara & 2010 & 2 & 43 & 0,37 & 2,78 \\
\hline Muda & & 7 & 150 & 1,28 & 5,09 \\
\hline
\end{tabular}

M: média do número de árvores por hectare; $\mathrm{S}$ desvio padrão do número de árvores por hectare.

A espécie teve regeneração natural abundante durante todo o período monitorado. Na primeira medição (1998), imediatamente após a exploração, foram registrados 427 indivíduos por hectare, sendo 21 varas e 406 mudas. O número de varas aumentou até o ano 2000, para 43 indivíduos por hectare e permaneceu constante até o final do período (2012).
O número de mudas aumentou após a exploração, porém 10 anos mais tarde (2007) sofreu uma redução de mais de $70 \%$ e assim permaneceu até o fim do monitoramento. Entretanto, apesar dessa redução, percebeu-se uma alta dinâmica no número de indivíduos em regeneração natural, permanecendo sempre em abundância na área. Neste caso, apesar de ser 
classificada como "em perigo de extinção" pelo MMA (2014), a espécie não corre o risco de ser extinta na área estudada.

\section{Conclusão}

A ausência de indivíduos em regeneração natural de Hymenaea parvifolia e Hymenolobium excelsum na área não explorada e a baixa densidade na área manejada indicam a dificuldade que essas espécies têm para regenerar e se estabelecer na floresta. E isso pode levá-las à extinção na floresta estudada. A regeneração natural de Vouacapoua americana é abundante, garantindo a sua permanência constante, sem correr o risco de extinção na área em estudo, embora esteja incluída na lista de espécies ameaçadas.

\section{Referências}

Almeida CM, Lima SF, Silva RCVM \& Gomes JI. 2001. Caracterização morfológica e anatômica de dez espécies de leguminosae ocorrentes em uma floresta tropical úmida localizada no município de Moju, Estado do Pará, p. 19-54. In: Silva JNM, Carvalho JOP de \& Yared JAG (eds.). A silvicultura na Amazônia Oriental: contribuições do projeto Embrapa/DFID. Belém: Embrapa Amazônia Oriental/DFID, 459p.

Braz EM, Carnieri C \& Arce JE. Um modelo otimizador para organização dos compartimentos de exploração em floresta tropical. Revista Árvore, 28(1): 77-83, 2004.

Carvalho, JOP. 1992. Struture and dynamics of a logged over Brazilian Amazonian rain forest. D. Phil. Thesis. OxfordUniversity,Oxford. 215p.

Carvalho, JOP, Silva JNM, Silva MG, Gomes JM, Taffarel M \& Nobre DNV. Mortality of girdled trees and survival of seedlings in canopy gaps after logging in an upland forest in the Brazilian Amazon. Revista de Ciências Agrárias, 56(1): 48-52, 2013.

Costa NSL, Jardim FCS, Gomes JM, Dionisio LFS \& Schwartz G. Responses in growth and dynamics of the shade-tolerant species Theobroma subincanum to logging gaps in the Eastern Amazon. Forest Systems, 29(1): 1-7, 2020.

Fernandes JM, Lopes CRAS, Ribeiro RS \& Silva DR. Leguminosae no acervo do herbário da Amazônia meridional, Alta Floresta, Mato Grosso. Enciclopédia Biosfera, 11(21): 2272-2293, 2015.

Ferreira GC, Hopkins MJG \& Secco RS. Contribuição ao conhecimento morfológico das espécies de leguminosae comercializadas no estado do Pará, como "angelim". Acta Amazonica, 34(2): 219-232, 2004a.

Ferreira GC, Gomes JI \& Hopkins MJG. Estudo anatômico das espécies de Leguminosae comercializadas no estado do Pará como "angelim". Acta Amazonica, 34(3): 387-398, 2004b.

Forget PM. Ten-Year Seedling Dynamics in Vouacapoua americana in French Guiana: A Hypothesis. Biotropica, 29(1): 124-126, 1997.

Gonzaga AL. Madeira: uso e conservação. Brasília: Instituto do Patrimônio Histórico e Artístico Natural, Cadernos Técnicos, 6, 246p, 2006.

Gourlet-Fleury S, Cornu G, Jésel S, Dessard H, Jourget JG, Blanc L \& Picard N. Using models to predict recovery and assess tree species vulnerability in logged tropical forests: A case study from French Guiana. Forest Ecology and Management, 209: 69-86, 2005.

Higman S, Mayers J, Bass S, Judd N \& Nusshaum R. 2015. Manual do manejo florestal sustentável. Tradução de Áurea Maria Brandi Nardelli. Viçosa: UFV. 398p.

IBGE (Instituto Brasileiro de Geografia e Estatística). 2012. Manual técnico da vegetação brasileira: Sistema fitogeográfico; inventário das formações florestais e campestres; técnicas e manejo de coleções botânicas; e procedimentos para mapeamentos. Rio de Janeiro: IBGE. 271p.

IBGE (Instituto Brasileiro de Geografia e Estatística). Pedologia: Mapa esquemático de solos. Estado do Pará, primeira edição, 2008. <ftp://geoftp.ibge. gov.br/informacoes_ambientais/pedologia/mapas/ unidades_da_federacao/pa_pedologia.pdf.> Acesso em: $17 / 1 \overline{1} / 20 \overline{17}$.

INMET (Instituto Nacional de Meteorologia). BDMEPBanco de Dados Meteorológicos para Ensino e Pesquisa. <http://www.inmet.gov.br/portal/index.php?r=bdmep/ bdmep > . Acesso em: 16/11/ 2017.

IUCN (International Union For Conservation Of Nature). 2012. IUCN Red List Categories and Criteria: Version 3.1. Gland, Switzerland and Cambridge, UK: IUCN. Second edition. 33p.

Jardim FCS \& Quadros LCL. Estrutura de uma floresta tropical dez anos após exploração de madeira em Moju, Pará. Rev. Ceres, Viçosa, 63(4): 427-435, 2016.

Jardim FCS. Natural regeneration in tropical forests. Revista de Ciências Agrárias, 58(1): 105-113, 2015.

Karsenty A \& Gourlet-Fleury S. Assessing Sustainability of Logging Practices in the Congo Basin's Managed Forests: The Issue of Commercial Species Recovery. Ecology and Society, 11(1): 1-26, 2006.

Kottek M, Grieser J, Beck C, Rudolf B \& Rubel F. World Map of the Köppen-Geiger climate classification 
updated. Meteorologische Zeitschrift, 15(3): 259-263, 2006.

LeãoNVM\& CarvalhoJOP.2001. Fenologiareprodutiva de 25 espécies arbóreas da Amazônia, p. 117-128 In: Silva JNM, Carvalho JOP \& Yared JAG (eds.). A silvicultura na Amazônia Oriental: contribuições do projeto Embrapa/DFID. Belém: Embrapa Amazônia Oriental/DFID. 458p.

Leão NVM, Ohashi ST, Freitas ADD, Nascimento MRSM, Shimizu ESC, Reis ARS, Galvão Filho AF \& Souza D. 2015. Colheita de sementes e produção de mudas de espécies florestais nativas. Belém: Embrapa Amazônia Oriental. Documentos 374, 47p.

Leite FS \& Rezende AV. Estimativa do volume de madeira partindo do diâmetro da cepa em uma área explorada de floresta amazônica de terra firme. Ciência Florestal, 20(1): 69-79, 2010.

Lopes JCA, Whitmore TC, Brown ND \& Jenning SB. 2001. Efeito da exploração florestal nas populações de mudas em uma floresta tropical úmida no município de Moju/PA, p. 227-251. In: Silva JNM, Carvalho JOP \& Yared JAG (Eds.). A silvicultura na Amazônia Oriental: contribuições do projeto Embrapa/DFID. Belém: Embrapa Amazônia Oriental/DFID. 459p.

Martins SV, Rodrigues RR, Gandolfi S \& Calegari L. 2012. Sucessão ecológica: Fundamentos e aplicações na restauração de ecossistemas florestais, p.21-42. In: Martins SV. Ecologia de florestas tropicais. 2 ed. Viçosa: Ed. UFV. 372p.

MMA (Ministério do Meio Ambiente). Instrução Normativa $\mathrm{n}^{\circ} 05$, de 11 de dezembro de 2006. Diário Oficial da União, 2006, 155p.

MMA (Ministério do Meio Ambiente). Portaria $n^{\circ} 443$, de 17 de dezembro de 2014. Diário Oficial da União, 2014, p. 110-121.

Mostacedo BC \& Fredericksen TS. Regeneration status of important tropical forest tree species in Bolivia: assessment and recommendations. Forest Ecology and Management, 124: 263-273, 1999.

Muñoz WA, Espinoza RT, Águila MYP, Vásquez LFÁ, Bardales LAM, Arévalo FR \& Gómez TP. Dinámica de la regeneración natural en claros y frecuencia de claros em bosques de varillal húmedo, Loreto, Perú. Conocimiento Amazónico. 1(1): 3-12, 2010.

Ngo TL \& Hölscher D. The fate of five rare tree species after logging in a tropical limestone forest (Xuan Son National Park, northern Vietnam). Journal Tropical Conservation Science, 7(2): 326-341, 2014.

Oliveira Junior RC \& Correa JRV. Caracterização dos solos do município de Belterra, Estado do Pará. Belém: Embrapa Amazônia Oriental. Documentos 88, 39p, 2001.
Oliveira LC. 1997. Dinâmica de crescimento e regeneração natural de uma floresta secundária no Estado do Pará, p. 69-87. In: Ecology and management of tropical secondary forest: Science people and policy, Costa Rica, Turrialba: CATIE/IUFRO/CIFOR/WWF/ GTZ. 233p.

Oliveira LC, Couto HTZ, Silva JNM \& Carvalho JOP. Exploração florestal e eficiência dos tratamentos silviculturais realizados em uma área de 136ha na Floresta Nacional do Tapajós, Belterra, Pará. Rev. Ciências Agrárias, 46: 195-213, 2006.

Quadros LCL. 2012. Dinâmica populacional de Pouteria gongrijpii Eyma (Abiurana) em clareiras de uma floresta tropical de terra firme, Moju, Pará. Dissertação (Mestrado em Ciências Florestais). Universidade Federal Rural da Amazônia. 71p.

Rabelo FG, Zarin DJ, Oliveira FA \& Jardim FCS. Regeneração natural de florestas estuarinas na região do rio Amazonas, Amapá, Brasil. Revista de Ciências Agrárias, 2(34): 129-138, 2000.

Reis LP, Carvalho JOP, Reis PCM, Gomes JM, Ruschel AR \& Silva MG. Crescimento de mudas de Parkia gigantocarpa Ducke em um sistema de enriquecimento em clareiras após a colheita da madeira. Ciência Florestal, 24(2): 431-436, 2014.

Salomão RP, Rosa NA \& Morais KAC. Dinâmica da regeneração natural de árvores em áreas mineradas na Amazônia. Bol. Mus. Para. Emílio Goeldi Ciências Naturais, 2(2): 85-139, 2007.

Salomão RP, Rosa NA, Nepstad DC \& Bakk A. Estrutura diamétrica e breve caracterização ecológica econômica de 108 espécies arbóreas da floresta amazônica brasileira. Interciencia, 20(1): 20-29, 1995.

Santos KF, Ferreira TS, Higuchi P, Silva AC, Vandresen PB, Costa A, Spada G, Schmitz V \& Souza F. Regeneração natural do componente arbóreo após a mortalidade de um maciço de taquara em um fragmento de floresta ombrófila mista em Lages/SC. Ciência Florestal, 25(1): 107-117, 2015.

Schwartz G, Falkowski V \& Peña-Claros M. Natural regeneration of tree species in the Eastern Amazon: Short-term responses after reduced-impact logging. Forest Ecology and Management, 385: 97-103, 2017.

Schwartz G, Lopes JCA, Mohren GMJ \& Peña-Claros M. Post-Harvesting silvicultural treatments in logging gaps: A comparison between enrichment planting and tending of natural regeneration. Forest Ecology and Management, 293: 57-64, 2013.

Silva BMS \& Cesarino F. Germinação de sementes e emergência de plântulas de jutaí (Hymenaea parvifolia Huber). Rev. Bras. Pl. Med. 18(1), supl. I, p. 256-263, 2016. 
Silva BMS, Lima JD, Dantas VAV, Moraes WS \& Sabonaro DZ. Efeito da luz no crescimento de mudas de Hymenaea parvifolia Huber. Revista Árvore, 31(6): 1019-1026. 2007.

Souza DV, Carvalho JOP, Mendes FS, Melo LO, Silva JNM \& Jardim FCS. Growth of Manilkara huberi and Manilkara paraensis after logging and silvicultural treatments in the municipality of Paragominas, Pará, Brazil. Floresta, 44(3): 485-496, 2014.
Vieira AH, Martins EP, Pequeno PLL \& Locatelli M. 2002. Interpretação fenológica de uma Floresta Ombrófila Aberta em Porto Velho/RO. Porto Velho: Embrapa CPAF. Boletim de pesquisa e desenvolvimento $12,13 p$.

Wadsworth FH \& Zweede JC. Liberation: acceptable production of tropical forest timber. Forest Ecology and Management, 233: 45-51, 2006.

\section{Biodiversidade Brasileira - BioBrasil. \\ Fluxo Contínuo}

n. 1, 2021

http://www.icmbio.gov.br/revistaeletronica/index.php/BioBR

Biodiversidade Brasileira é uma publicação eletrônica científica do Instituto Chico Mendes de

Conservação da Biodiversidade (ICMBio) que tem como objetivo fomentar a discussão e a disseminação de experiências em conservação e manejo, com foco em unidades de conservação e espécies ameaçadas.

ISSN: $2236-2886$ 